\title{
Revisiting the condition-dependence of melanin-based plumage
}

\author{
Sarah Guindre-Parker and Oliver P. Love \\ S. Guindre-Parker(slg2154@columbia.edu) and O. P. Love, Dept of Biological Sciences, Univ. of Windsor, Windsor, ONN9B 3P4, Canada. \\ Present address for SGP: Dept of Ecology, Evolution and Environmental Biology, Columbia Univ., 1200 Amsterdam Avenue, New York, \\ NY 10027, USA.
}

\begin{abstract}
Support against the condition-dependence of melanin plumage signals has relied on data from species exhibiting both melanin- and carotenoid-based plumage ornaments. As the mechanisms leading to variation in carotenoid- and melaninbased plumage differ fundamentally, these systems may not be ideal to assess the condition-dependence of melanin signals. Instead, we hypothesized that melanin-plumage is more likely to signal condition in purely achromatic species. We performed a meta-analysis reviewing evidence for condition-dependent melanic plumage: we compared the net effect size for the relationship between melanin traits and condition in species that are achromatic versus species that also display a carotenoid-based trait. Our results indicate that melanin plumage is condition-dependent in species of both plumage types. Contrarily to our prediction, this finding suggests that melanin ornament condition-dependence is not conditional on the context of other ornaments within a species. Instead, melanin ornaments should be viewed as potential conditiondependent signals in all species.
\end{abstract}

Condition-dependent ornaments are those for which an individual's condition (either current, or at the time of feather growth) correlates with the quality of its plumage ornament. There has been great interest in the evolution of condition-dependent plumage signalling (Hill 2011), at least partly because condition-dependent plumage production mechanisms remain unresolved. As such, a particularly debated topic is whether melanin-based plumage may act in a condition-dependent manner. Several studies have generalized that black plumage is not costly to produce or maintain, and is thus not condition-dependent primarily because studies have failed to detect correlations between ornaments and condition in their study systems (Hill and Brawner 1998, Badyaev and Hill 2000, McGraw and Hill 2000, Senar et al. 2003). However, support against the condition-dependence of melanin-based signals has relied on data from species displaying simultaneously melanin- and carotenoid-based plumage ornaments (Badyaev and Hill 2000, McGraw and Hill 2000, Senar et al. 2003, Hill et al. 2009). Authors justify that these within-species comparisons are ideal for understanding the use of melanin- and carotenoid-based signals. The issue with this approach lies in the significantly different mechanisms through which carotenoid- and melanin-based signals are produced in birds: carotenoid pigments must be acquired through the diet while melanin pigments are produced de novo (reviewed by Griffith et al. 2006). Inherently, this difference makes carotenoid-based ornaments more likely to relate to variation in diet quality and co-vary with condition whereas the relationship between melanin-based signals and condition remains less evident. It is also possible that multiple ornaments trade-off with one another such that enhancing the expression of one plumage trait results in a lower quality ornament for the other (as with melanin and carotenoid signals; Alonso-Alvarez and Galván 2011). Lastly, multiple ornament theory does not predict the simultaneous evolution of two condition-related ornaments; instead multiple ornaments should signal different aspects of individual quality (Møller and Pomiankowsky 1993). As such, previous studies on species possessing both carotenoid- and melaninbased signals may have negatively biased support for the condition-dependence of melanin- relative to carotenoid-based plumage (although see Parker et al. 2003). Recent findings in species that lack any carotenoid plumage ornaments suggest that black plumage may indeed be condition-dependent (Gladbach et al. 2011, Guindre-Parker et al. 2013a). We hypothesized that melanin-based ornaments are more likely to serve as reliable indicators of condition in species that are achromatic compared to ones that also have a carotenoidbased trait. To examine this possibility, we performed a meta-analysis to test whether the condition-dependence of melanin-based plumage is stronger in achromatic species compared to species that display both carotenoid and melanin ornaments.

\section{Revisiting the literature}

We surveyed published studies on condition-dependent melanin plumage by searching Web of Science for studies that included 'black plumage' or 'melanin plumage', in 
combination with the terms 'condition' or 'quality'. Additionally, we surveyed the references of previous metaanalyses on melanin-based plumage for studies that also contained information on individual condition (Jawor and Breitwisch 2003, Roulin 2009, Meunier et al. 2011). We characterized species as either achromatic (primarily black, brown, grey or white) or chromatic (also possessing a carotenoid-based red, orange or yellow ornament) to investigate whether the presence of a carotenoid-based signal can influence the condition-dependence of a melanin signal. We excluded species with iridescent plumage as it is primarily structurally based (Shawkey et al. 2006), as well as because it is difficult to determine how birds perceive iridescence without more complex visual modeling. Lastly, we excluded studies from which we could not extract effect size information.

We performed a random effects meta-analysis using correlation coefficients $(r)$ as the effect size when reported; otherwise, we used appropriate formulae to calculate $r$ from other test statistics (as in Boncoraglio and Saino 2007). We calculated the weighted mean if multiple effect sizes existed for the same species, such that each species is represented only once in our meta-analysis (as in Boncoraglio and Saino 2007). We defined a positive effect size to indicate studies that found larger or darker melanin-based plumage to relate to better condition (i.e. better immune parameters, greater body condition or mass). Given our criteria, we identified 26 suitable studies that included 14 achromatic and 7 chromatic species (Supplementary material Appendix 1, Table A1). We calculated the summary effect size (a weighted mean) in $\mathrm{R}$ (ver. 2.14.2) using the package metacor, which standardizes effect sizes by converting $r$ to Fisher's Z. We calculated a pooled summary effect size for all species, as well as separate summary effect sizes for achromatic and chromatic species; we compared the summary effect size for achromatic and chromatic species using a two-tailed t-test. Lastly, we calculated the correlation between standardized effect size and sample size using spearman's rank to determine whether a publication bias exists.

\section{Condition-dependent melanin signals}

Our review of the literature suggests that current conclusions on the lack of a relationship between melanin-based plumage and condition may need updating; we found support for a significant positive relationship between condition and melanin plumage (pooled achromatic and chromatic species; $r=0.23 \pm 0.05$ and $p=0.00006$ ) where darker or larger areas of melanin plumage signal higher condition (Fig. 1). This relationship remained significant in both achromatic species and species that also displayed a carotenoid ornament $(\mathrm{p}<0.0002)$, and there was no significant difference between the mean effect size for achromatic versus chromatic species $(t=0.48, \mathrm{DF}=18.4, \mathrm{p}=0.64)$. This finding does not support previous conclusions that melanin-based plumage is not condition-dependent (as in Badyaev and Hill 2000, Senar et al. 2003). Furthermore, this result refutes our hypothesis that melanin plumage condition-dependence differs between species that also have or lack a carotenoid signal.

Signal context can influence how receivers interpret a signal (territory location: Höglund et al. 1994; age: Badyaev and

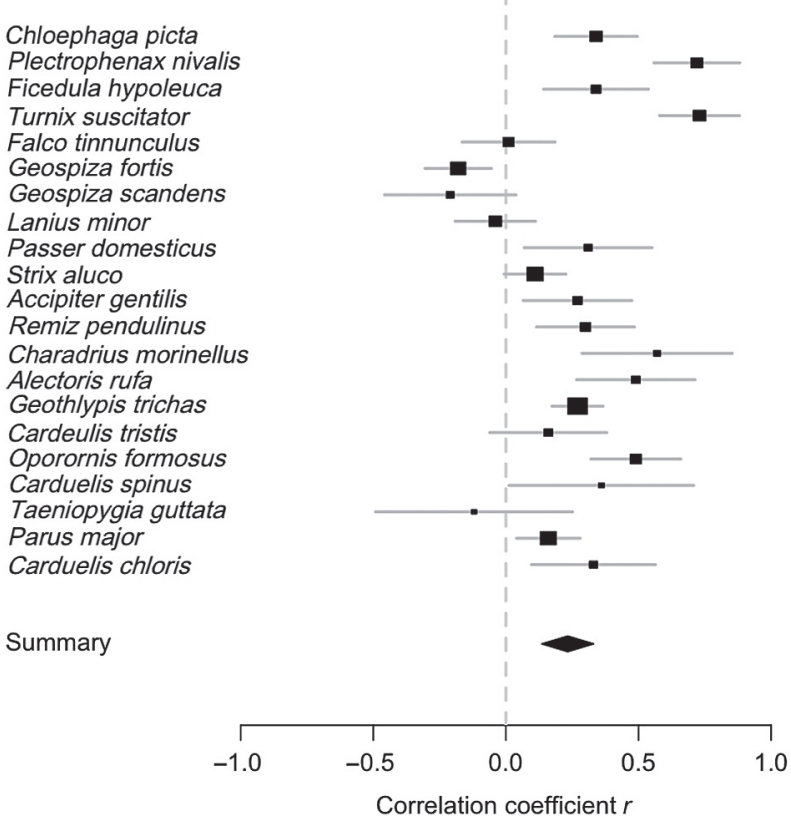

Figure 1. Effect sizes for the relationship between melanin plumage ornaments and individual condition. Each species' scientific name is indicated to the left of the figure, with boxes indicating the effect size (correlation coefficient $r$ ) and the whiskers indicating the standard error for each study. The size of boxes reflects studies' relative sample sizes. The summary effect is indicated by a diamond at the bottom of the plot, where $r=0.23 \pm 0.05$ and $\mathrm{p}=0.00006^{*}$.

Duckworth 2003), and this context can include additional ornaments displayed by a species. Specifically, researchers are well aware that species can use multiple ornaments and that the use of a plumage signal may be relative to the other ornaments present in a species (Møller and Pomiankowsky 1993). However, we found that melanin-based signals can act as condition-dependent ornaments regardless of whether or not species possess a carotenoid signal. While multiple ornaments can influence each other, even in achromatic species (Guindre-Parker et al. 2013b), our meta-analysis suggests that multiple ornaments do not affect the likelihood that melanin-based plumage will be condition-dependent.

While the number of articles considered in our metaanalysis is low, studies examining the condition-dependence of achromatic plumage are currently limited. There was a significant negative correlation between standardized effect size and sample size (spearman's rank: $r h o=-0.63, \mathrm{n}=26$, $\mathrm{p}=0.0052$ ), suggesting that studies with negative effects (in opposite direction of predictions) or no effect are less likely to be published than ones with the predicted positive relationship between condition and melanin plumage. This perceived bias might be enhanced because published studies that failed to detect a relationship between melanin plumage and condition often did not report statistical results and were excluded from our analysis. Evidence for a publication bias may also suggest that researchers have been discouraged from pursuing or publishing studies on the conditiondependence of black plumage. However, despite the limited publications that were available to be included in our analysis, we detected a net significant positive relationship between melanin plumage and condition. While interpreting our results in 
light of the publication bias is challenging, our findings refute the notion that melanin-based plumage is not condition-dependent. Studies on the condition-dependence of black plumage have been scarce relative to carotenoid signals yet we are hopeful that our results will encourage future research to explore the potential condition-dependence of achromatic plumage across a variety of contexts before generalizing on how achromatic plumage may or may not relate to individual condition.

At this time, few studies have performed experimental manipulations (i.e. manipulated condition) and studies included in our analysis are largely correlative (although see McGraw and Hill 2000). This limits our understanding of how condition causally mediates melanin ornament quality: for example, melanin ornaments may signal social rank, with dominant individuals also being in better condition. Piault et al. (2012) recently manipulated body condition and showed that individuals in better body condition produced larger black bands on their tail feathers; we urge researchers to consider manipulating condition in future studies as well.

\section{Costs of melanin-based plumage}

Our understanding of the mechanisms underlying the production and condition-dependence of carotenoid-based plumage has advanced more rapidly than for melanin-based plumage (McGraw 2006). The costs of producing black feathers remain largely unresolved (Hill 2011), and this has also likely contributed to the perception that these ornaments cannot signal condition.

While the costs of melanin production are largely unresolved, the process of melanogenesis is well documented (Prota 1992). Melanocytes are specialized melanin-producing cells derived from embryonic neural crest cells that migrate to the epidermis during development; here they differentiate and begin synthesizing melanin. The activity of melanocytes is chiefly regulated by melanocortin 1 receptors, membrane protein receptors that bind to melanocortin hormones (Ducrest et al. 2008). Melanin is produced de novo by melanosomes (small organelles found within melanocytes) from the semi-essential amino acid tyrosine. Tyrosine is converted to the intermediate dopaquinone, catalyzed by the rate-limiting enzyme tyrosinase; dopaquinone can then be converted to pheomelanin or eumelanin (if combined with the non-essential amino acid cysteine versus converted to leucodopachrome, respectively). These two melanin-pigment types lead to different color phenotypes: high concentrations of eumelanin cause dark, black coloration in feathers while pheomelanin leads to buffy brown colors (McGraw 2008). Once melanosomes have produced melanin pigments, they migrate to the cell membrane in order to incorporate the pigment into feathers or skin. The numerous steps of melanogenesis show potential for condition-dependence, and although little empirical work has explored these possibilities, we speculate on these processes to identify promising areas of research.

1) Melanogenesis is under genetic control and dependent on cellular signals, such that melanin-production is dependent on the cellular environment. As an example, the mobilization and differentiation of melanocytes during development is susceptible to developmental stressors, which could ultimately alter the density and position of melanocytes within the epidermis (discussed in McGraw 2006). Similarly, incorporating melanin into feathers requires the intra-cellular movement of melanins, which is dependent on motor proteins that are sensitive to cellular physiological conditions.

2) Tyrosine can both be obtained through the diet, or produced from the essential amino acid phenylalanine (which must be obtained exogenously). These amino acids have the potential to be limiting depending on the quality and quantity of various dietary items consumed (Poston et al. 2005). This possibility has been highlighted previously yet few studies have examined this possibility (McGraw 2008).

3) Tyrosinase activity is the rate-limiting step in melanogenesis (Prota 1992), such that regulators of this enzyme are crucial to the production of melanin. This enzyme requires mineral co-factors (i.e. copper, calcium), which are acquired through the diet and have the potential to be limiting (discussed in McGraw 2008). Any other factors enhancing or reducing tyrosinase activity would have the potential to influence melanogenesis.

4) Genes regulating melanocortin 1 receptors can have pleiotropic effects on immune function and glucocorticoid stress responses (Ducrest et al. 2008), linking melanin ornament production and individual variation in condition.

5) A variety of hormones, which themselves may be condition-dependent, can affect melanogenesis (e.g. luteinizing hormone stimulates the activity of tyrosinase: Hall 1969). Increased glucocorticoids have been shown to reduce the concentration of pheomelanin in plumage, potentially through a negative feedback loop with melanocortins (Roulin et al. 2008). Hormonal effects on melanogenesis have implications for the role that maternal effects (maternal hormones transferred through the yolk; Love et al. 2013) may play in affecting offspring melanin phenotype.

While we lack a thorough understanding of how melanin synthesis may directly or indirectly vary with condition, evidence from ecological studies linking a variety of condition proxies and melanin ornaments is accumulating. Melaninbased plumage correlates with body condition (Parejo et al. 2011), survival (Griffith 2000), oxidative balance (Galván and Alonso-Alvarez 2008), immunity (Guindre-Parker et al. 2013b), social rank (McGraw et al. 2003), and diet quality (McGlothlin et al. 2007). Although challenging, investigating the biochemical links between melanin ornaments and proxies of individual condition will be essential to furthering our understanding of how and when melanin-ornaments reliably signal condition (suggested in Hill 2011). Integrating across disciplines (i.e. biochemistry, cellular biology) may further help behavioural ecologists to apply new methods for understanding these mechanisms (McGraw 2008). We would argue that tackling mechanism-based questions might be more feasible in achromatic species where the mechanism of plumage production is relatively simpler than species that display multiple signals produced by different mechanisms. Species with melanin-based colour polymorphisms may also provide a useful tool for the study of melanogenesis. 


\section{Conclusion}

Despite being an ancestral trait to coloured plumage (Stoddard and Prum 2011), purely achromatic plumage has been retained in multiple species both within and across avian families. Furthermore, black plumage can be a highly conspicuous and efficient mode of visual communication, particularly when contrasting black and white plumage patches are adjacent to one another (Endler 1992); melanin signals can also act to amplify other ornaments (Brooks 1996). In certain environments, black plumage signals may also be more contrasting relative to their background, making them easier to detect and assess (Wiley 2006). Together, this suggests that in a multitude of species, coloured plumage is not necessary to serve in effective visual communication. Furthermore, our review of the literature suggests that melanin-based plumage may signal condition even when species also display a carotenoid ornament. While the specific mechanisms linking black plumage production and condition remain unresolved, empirical studies reviewed herein have illuminated a multitude of potential costs that may enforce the condition-dependence of black plumage. Further studies on the function of melanin ornaments both in species with and without additional coloured plumage signals will be necessary to determine how black plumage functions in different contexts. These studies will be imperative in understanding how the diversity of plumage colours and patterns observed in birds has evolved.

Acknowledgements - This work was supported by an operating grant from the Natural Science and Engineering Research Council of Canada to OPL. Additional funds for this research included graduate awards from the Natural Science and Engineering Research Council of Canada, the Ontario Ministry of Training, Colleges and Universities, as well as the Univ. of Windsor to SGP.

\section{References}

Alonso-Alvarez, C. and Galván, I. 2011. Free radical exposure creates paler carotenoid-based ornaments: a possible interaction in the expression of black and red traits. - PLoS One 6: e19403.

Badyaev, A. V. and Hill, G. E. 2000. Evolution of sexual dichromatism: contribution of carotenoid- versus melanin-based coloration. - Biol. J. Linn. Soc. 69: 153-172.

Badyaev, A. V. and Duckworth, R. A. 2003. Context-dependent sexual advertisement: plasticity in development of sexual ornamentation throughout the lifetime of a passerine bird. - J. Evol. Biol. 16: 1065-1076.

Boncoraglio, G. and Saino, N. 2007. Habitat structure and the evolution of bird song: a meta-analysis of the evidence for the acoustic adaptation hypothesis. - Funct. Ecol. 21: 134-142.

Brooks, R. 1996. Melanin as a visual signal amplifier in male guppies. - Naturwissenschaften 83: 39-41.

Ducrest, A.-L., Keller, L. and Roulin, A. 2008. Pleiotropy in the melanocortin system, coloration and behavioural syndromes. - Trends. Ecol. Evol. 23: 502-510.

Endler, J. A. 1992. Signals, signal condition, and the direction of evolution. - Am. Nat. 139: 125-153.

Galván, I. and Alonso-Alvarez, C. 2008. An intracellular antioxidant determines the expression of a melanin-based signal in a bird. - PLoS One 3: e3335.
Gladbach, A., Gladbach, D. and Quillefeldt, P. 2011. Male achromatic wing colouration is related to body condition and female reproductive investment in a dichromatic species, the upland goose. - J. Ethol. 29: 243-249.

Griffith, S. C. 2000. A tradeoff between reproduction and a condition-dependent sexually selected ornament in the house sparrow Passer domesticus. - Proc. R. Soc. B 267: 1115-1119.

Griffith, S. C., Parker, T. H. and Olson, V. A. 2006. Melanin- versus carotenoid-based sexual signals: is the difference really so black and red? - Anim. Behav. 71: 749-763.

Guindre-Parker, S., Gilchrist, H. G., Baldo, S. and Love, O. P. 2013a. Alula size signals male condition and predicts reproductive performance in an Arctic-breeding passerine. J. Avian Biol. 44: 209-215.

Guindre-Parker, S., Gilchrist, H. G., Baldo, S., Doucet, S. M. and Love, O. P. 2013b. Multiple achromatic plumage ornaments signal to multiple receivers. - Behav. Ecol. 24: 672-682.

Hall, P. F. 1969. Hormonal control of melanin synthesis in birds. - Gen. Comp. Endocrinol. 2: 451-458.

Hill, G. E. 2011. Condition-dependent traits as signals of the functionality of vital cellular processes. - Ecol. Lett. 14: 625-634.

Hill, G. E. and Brawner, W. R. III 1998. Melanin-based plumage coloration in the house finch is unaffected by coccidial infection. - Proc. R. Soc. B 265: 1105-1109.

Hill, G. E., Hood, W. R. and Huggins, K. 2009. A multifactorial test of the effects of carotenoid access, food intake and parasite load on the production of ornamental feathers and bill coloration in American goldfinches. - J. Exp. Biol. 212: $1225-1233$.

Höglund, J., Alatalo, R. V., Lundberg, A. and Rätti, O. 1994. Context-dependent effects of tail-ornament damage on mating success in black grouse. - Behav. Ecol. 5: 182-187.

Jawor, J. M. and Breitwisch, R. 2003. Melanin ornaments, honesty, and sexual selection. - Auk 120: 249-265.

McGlothlin, J. W., Duffy, D. L., Henry-Freeman, J. L. and Ketterson, E. D. 2007. Diet quality affects an attractive white plumage pattern in dark-eyed juncos (Junco hyemalis). - Behav. Ecol. Sociobiol. 61: 1391-1399.

McGraw, K. J. 2006. Chapter 5. Mechanics of melanin-based coloration. - In: Hill, G. E. and McGraw, K. J. (eds), Bird colouration - mechanisms and measurements. Harvard Univ. Press, pp. 177-243.

McGraw, K. J. 2008. An update on the honesty of melaninbased color signals in birds. - Pig. Cell. Melano. Res. 21: 133-138.

McGraw, K. J. and Hill, G. E. 2000. Differential effects of endoparasitism on the expression of carotenoid- and melanin-based ornamental coloration. - Proc. R. Soc. B 267: 1525-1531.

McGraw, K. J., Dale, J. and Mackillop, E. A. 2003. Social environment during molt and the expression of melanin-based plumage pigmentation in male house sparrows (Passer domesticus). - Behav. Ecol. Sociobiol. 53: 116-122.

Meunier, J., Figueiredo Pinto, S., Burri, R. and Roulin, A. 2011. Eumelanin-based coloration and fitness parameters in birds: a meta-analysis. - Behav. Ecol. Sociobiol. 65: 559-567.

Møller, A. P. and Pomiankowsky, A. 1993. Why have birds got multiple sexual ornaments? - Behav. Ecol. Sociobiol. 32: 167-176.

Parejo, D., Silva, N., Danchin, E. and Aviles, J. M. 2011. Information content of melanin-based plumage colour in adult Eurasian kestrels. - J. Avian. Biol. 42: 49-60.

Piault, R., van den Brink, V. and Roulin, A. 2012. Conditiondependent expression of melanin-based coloration in the Eurasiam kestiel. - Naturwissenschaften 99: 391-396. 
Poston, J. P., Hasselquist, D., Stewart, I. R. L. and Westneat, D. F. 2005. Dietary amino acids influence plumage traits and immune responses of male house sparrows, Passer domesticus, but not as expected. - Anim. Behav. 70: 1171-1181.

Prota, G. 1992. Melanins and melanogenesis. - Academic Press.

Roulin, A. 2009. Covariation between eumelanic pigmentation and body mass only under specific conditions. - Naturwissenschaften 96: 375-382.

Roulin, A., Almasi, B., Rossi-Pedruzzi, A., Ducrest, A.-L., Wakamastu, K., Miksik, I., Blount, J. D., Jenni-Eiermann, S. and Jenni, L. 2008. Corticosterone mediates the conditiondependent component of melanin-based coloration. - Anim. Behav. 75: 1351-1358.

Supplementary material (Appendix JAV-00190 at <www. oikosoffice.lu.se/appendix $>$ ). Appendix 1 .
Senar, J. C., Figuerola, J. and Domenech, J. 2003. Plumage coloration and nutritional condition in the great tit Parus major: the roles of carotenoids and melanins differ. - Naturwissenschaften 90: 234-237.

Shawkey, M. D., Hauber, M. E., Estep, L. K. and Hill, G. E. 2006. Evolutionary transitions and mechanisms of matte and iridescent plumage coloration in grackles and allies (Icteridae). J. R. Soc. Interface 3: 777-786.

Stoddard, M. C. and Prum, R. O. 2011. How colorful are birds? Evolution of the avian plumage color gamut. - Behav. Ecol. 22: 1042-1052.

Wiley, R. H. 2006. Signal detection and animal communication. - Adv. Stud. Behav. 36: 217-247. 